\title{
SULLA RACCOLTA DELLE SOSTANZE CHE ESALANO DURANTE LA RIFUSIONE DELLE ROCCE ERUTTIVE
}

\author{
G. Ponte
}

Nel 1923 presentai alla XII riunione della Società per il Progresso delle Scienze una relazione sui progressi della vulcanologia $\left({ }^{1}\right.$ ). Fra l'altro esposi che le ricerche sperimentali fatte sul terreno, specialmente durante le eruzioni, avevano fatto progredire considerevolmente questa scienza, tanto da farmi azzardare a dire che un giorno avremmo potuto domare le forze distruttrici dei vulcani.

Ciò che un quarto di secolo addietro sembrava arduo solo pensare oggi è una realtà. Già l'uomo è riuscito a deviare $i$ torrenti di lava; la terribile colata del Mauna Loa che nel 1936 stava per distruggere la città di Hilo nell'isola Hawaii, fu arrestata per averne deviato il corso con un bombardamento alle bocche effusive. Sull'Etna non è stato fatto alcun tentativo del genere perché è mancata un'organizzazione di difesa, ma ora con decreto prefettizio è stato costituito un Comitato per la difesa contro le calamità vulcaniche, il quale con la collaborazione dell'Istituto Nazionale di Geofisica sta organizzando un servizio completo di osservazioni vulcanologiche e geofisiche.

L'Istituto Vulcanologico Etneo, con lalsoratorio in Catania nel palazzo delle scienze dell'Università e stazioni di osservazione sull'Etna, fondato con il contributo degli Enti locali allo scopo precipuo di difendere la popolazione etnea dalle calamità vulcaniche, ha esteso la sua attività anche alle ricerche puramente scientifiche con risultati di notevole importanza, malgrado gli scarsi mezzi sinora avuti a disposizione. All'8n riunione dell'Association de Volcanologie de l'Union Géodésique et Géophysique internationale, tenutasi ad Oslo nell'agosto del $19 ! 8$ presentai un riassunto delle principali ricerche fatte sull'Etna durante le recenti eruzioni. Esporrò in questa breve nota un nuovo metodo di ricerche, iniziato quest'anno, appena mi è stato possibile avere $\mathrm{i}$ mezzi.

(1) G. Ponte, Nuovi metodi e risultati nelle ricerche sul vulcanismo. Atti Soc. It. Progresso Scienze, XII Riunione 1923. Roma. 
Dopo mezzo secolo ho potuto riprendere le esperienze di Gautier, di Chamberlin e di Brun non più continuate da altri studiosi.

A. Brun fondeva le vulcaniti e raccoglieva nel vuoto le sostanze volatili che da esse esalavano. Io Sondo le rocce laviche dell'Etna in un crogiuolo tubolare di platino, dentro il quale faccio circolare una corrente di gas inerte preferibilmente argo. La ditta I. Staccioli di Roma mi ha costruito a tal nopo un crogiuolo lungo $360 \mathrm{~mm}$ del diametro di $15 \mathrm{~mm}$ chiuso ad un estremo e all'altro munito di turacciolo al quale sono saldati due tubicini del diametro di $3 \mathrm{~mm}$; uno di

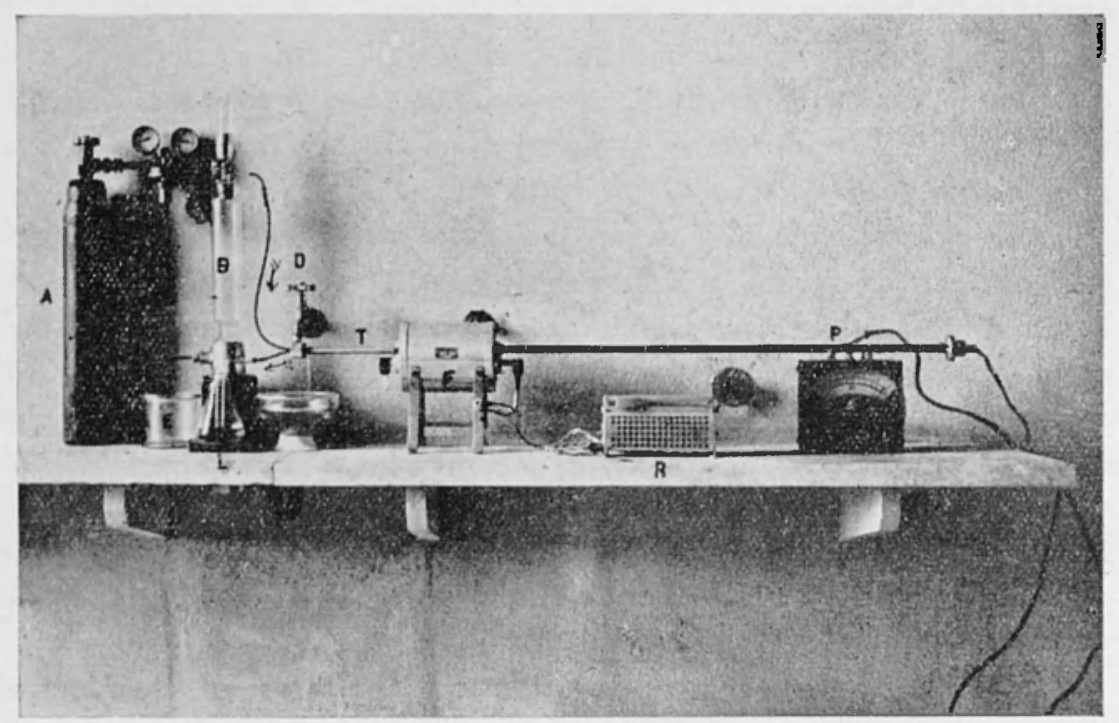

Fig. I - A: bombola con argo e manometro per riempire il propulsatore B; B: propulsatore a caduta di mercurio; $\mathrm{T}$ : tubo di platino per meta nel forno; $\mathrm{F}$ : forno tubolare elettrico; P: pirometro con coppia introdotta nel forno F; L: boccia di lavaggio del gas che esce dal tubo $\mathrm{T}$; $\mathrm{D}$ : getto d'acqua per refrigerare la testa del tubo T; E: vaso per lo scarico del mercurio del propulsatore $B$

essi è lungo quasi quanto il crogiuolo, l'altro sporge solo all'esterno e serve per l'uscita del gas inalato nel crogiuolo attraverso il tubicino lungo durante la fusione della roccia.

La quantitì di roccia lavica che viene sottoposta alla fusione è di circa $10 \mathrm{gr}$; essa viene polverizzata e pesata e poi, posta in una navicella di platino, viene introdotta fino in fondo al crogiuolo. Il tubicino lungo, attaccato al coperchio, viene introdotto in fondo fino a chiudere il crogiuolo. Ai due tubicini che sporgono fuori del coperchio s'innestano dei tubi di caucciù e precisamente a quello che si 
prolunga dentro il crogiuolo si attacca il tubo che viene dal propulsatore d'argo; l'altro tubo di caucciù collega il tubicino corto di platino con una boccia di lavaggio per trattenere le eventuali tracce dei sali volatili che potrebbero esser trascinati dalla debole corrente gassosa. Tutto il coperchio del crogiuolo viene rivestito di paraffina e refrigerato con un getto d'acqua durante la fusione per aiutare la condensazione dei sali volatili che avviene quasi totalmente nel tratto fredllo del tubo di platino.

La fusione delle vulcaniti elnee avviene dopo circa 15 minuti, appena il forno tubolare, ove è introdotto il crogiuolo, raggiunge la temperatura di $1150^{\circ}$. Le sostanze volatili dopo circa 20 minuti esalano tutte dalla roccia in fusione.

In fine, spento il forno e raffreddato il crogiuolo, si apre il coperchio e si tira fuori il lubicino lungo, avendo cura di lavarlo con acqua distillata; lo stesso si fa per il crogiuolo e la navicella ove trovasi la roccia già solidificata. La soluzione salina che si ricava dal lavaggio serve per l'analisi quantitativa dei cloruri che generalmente non raggiungono i 4 milligrammi per ogni $10 \mathrm{gr}$ di roccia fusa. Questa viene analizzata con il metodo spettrografico per stabilire in quali rapporti si trovino $i$ vari cloruri.

La raccolta delle sostanze volatili che esalano dalle vulcaniti rifuse con questo metodo della circolazione di un gas inerte, offre pure la possibilità di studiare particolari reazioni delle vulcaniti in fusione, introducendo assieme al gas inerte opportuni reattivi.

Catania - Istituto di Vulcanologia dell'Università - Dicembre 1949. 